\title{
APLICACIÓN Y ANÁLISIS DE UN MODELO PARA LA PREDICCIÓN DE LA EVAPOTRANSPIRACIÓN DEL CULTIVO (ETC) EN CEBOLLA Y MAÍZ EN CONDICIONES SEMIÁRIDAS
}

\author{
Barroso Costa, F. R. ${ }^{1}$ Ortega Álvarez, J. F. ${ }^{2}$ Ballesteros González, R. $^{3}$
}

\begin{abstract}
1 Ingeniero Agrónomo, Estudiante de doctorado, Centro Regional de Estudios del Agua (CREA), Universidad de Castilla-La Mancha, Ctra. de Las Peñas km. 3.4, 02071 Albacete, fellyperodrigo.barrosocosta@alu.uclm.es.

${ }^{2}$ Profesor Titular, Centro Regional de Estudios del Agua (CREA), Universidad de Castilla-La Mancha, Ctra. de Las Peñas km. 3.4, 02071 Albacete, jose.ortega@uclm.es.

${ }^{3}$ Personal Investigador, Centro Regional de Estudios del Agua (CREA), Universidad de Castilla-La Mancha, Ctra. de Las Peñas km. 3.4, 02071 Albacete, rocio.ballesteros@uclm.es.
\end{abstract}

\section{Resumen}

La mejora de la gestión de los recursos hídricos en la agricultura es un objetivo prioritario a nivel mundial. Los modelos de predicción de evapotranspiración del cultivo (ETc) integrados en el asesoramiento al regante ayudan en la mejora de la eficiencia de uso del agua. La predicción de datos climáticos es muy útil en la estimación de la evapotranspiración de referencia (ETo) en tiempo real para optimizar la programación del riego. Así, el objetivo de este trabajo es analizar los resultados sobre la ETo y ETc, para los cultivos de maíz y cebolla, a partir del uso de las predicciones ofrecidas por la Agencia Estatal de Meteorología (AEMET) y la estimación de la ETo mediante las Redes neuronales Artificiales (RNA) en dos ubicaciones semiáridas localizadas en La Mancha Oriental. En el cálculo de ETo a través de la fórmula de PM-56 fueron utilizados datos observados diarios de dos estaciones meteorológicas localizadas en La Mancha Oriental (Pozo Cañada y Tarazona de La Mancha) para los años de 2011 y 2012. También fue estimada la ETo a través de la metodología de RNAs, utilizando el software FORETo (Ballesteros, 2014) utilizando las variables climáticas predichas por la AEMET. A partir de los valores predichos de ETo fue posible obtener los valores de ETc en los 7 días siguientes. Estas predicciones fueron comparadas con los datos de ETc observados para el mismo periodo. Los resultados estadísticos indican que los valores predichos de ETc, de forma general, se ajustan adecuadamente a los valores observados en los 7 días de predicción. Los mejores valores de ajuste se observan durante los primeros días de predicción $\left(R^{2}=0.95\right)$. A pesar de haber una disminución en el ajuste en los últimos días de predicción $\left(R^{2}=0.92\right)$, el modelo se mantiene dentro del rango establecido para un buen ajuste. Así se concluye que las predicciones presentan un buen ajuste en relación a los datos observados en las estaciones, lo que demuestra que el modelo puede ser utilizado como una herramienta adicional de ayuda a los regantes, que podrán tener una predicción de 7 días con una gran fiabilidad de los datos.

\section{1 - Introducción}

La mejora de la gestión de los recursos hídricos en la agricultura es un objetivo prioritario a nivel mundial y especialmente en los territorios semiáridos de la cuenca mediterránea. 
Una forma de mejorar la gestión de unos recursos hídricos escasos es aumentar la eficiencia de uso del agua, haciendo inversiones a nivel de conocimiento y capacidad humana (Comprehensive Assessment of Water Management in Agriculture, 2007), ya sea en las áreas urbanas o en el ámbito rural, ya que en las zonas semiáridas el consumo mayoritario del agua es en regadío, y en algunos casos ese consumo va por encima del $90 \%$ de los usos consuntivos totales (MARM, 2010), como es el caso de La Mancha Oriental. Los modelos de predicción de evapotranspiración del cultivo (ETc) ayudan en la mejora esta eficiencia, pero se hacen necesarios modelos que puedan predecir la ETc diaria en un periodo de tiempo suficientemente amplio para su utilización práctica por los regantes en la determinación de las necesidades hídricas de los cultivo.

La predicción de datos climáticos es muy útil para la estimación de la evapotranspiración de referencia (ETo) en tiempo real para optimizar la programación del riego. El servicio de predicción de la Agencia Estatal de Meteorología (AEMET) (http://www.aemet.es/es/eltiempo/prediccion) proporciona pronósticos diarios de variables climáticas como la temperatura máxima (TMAX) y mínima (TMIN), humedad relativa $(\mathrm{Hr})$ y velocidad del viento (W) en una base semanal utilizando las entradas generadas por el sistema de predicción por conjuntos del European Centre for Medium-Range Weather Forecast (ECMWF) (http://www.ecmwf.int/). La AEMET también ofrece los registros de las variables climáticas observadas. Estas predicciones se basan en las perturbaciones atmosféricas sobre su estado inicial mediante la aplicación y modelos complejos para la predicción a medio plazo (3 a 7 días) (ECMWF, 2015). Para el cálculo de ETc se utiliza como referencia el valor de evapotranspiración de referencia (ETo) obtenido a través de la fórmula propuesta por Penman-Monteith (PM-56) (Allen et al., 1998). También se puede estimar mediante modelos basados en Redes Neuronales Artificiales (RNA), como una alternativa a los modelos más tradicionales en un contexto de limitación en la disponibilidad de datos meteorológicos (Ballesteros, 2014).

El objetivo de este trabajo es analizar los resultados sobre la ETo y ETc, para los cultivos de maíz y cebolla, del uso de modelos de predicción semanal basado en los pronósticos de la AEMET y en la estimación de la ETo mediante RNA en dos ubicaciones semiáridas localizadas en La Mancha Oriental.

\section{2 - Material y métodos}

En el cálculo de ETo a través de la fórmula de PM-56 fueron utilizados datos diarios de dos estaciones meteorológicas localizadas en La Mancha Oriental (Pozo Cañada y Tarazona de La Mancha). Los datos meteorológicos observados para los años de estudio (2011 y 2012), se obtuvieron a través del Sistema de Información Agroclimática para el Regadío (SIAR). Los datos diarios de predicción semanal para los mismos años de estudio se obtuvieron, diariamente, de la AEMET para la estimación de la ETo predicha a través de la metodología de RNAs, utilizando el software FORETo, que fue calibrado y validado con datos diarios predichos de TMAX y TMIN para las mismas estaciones meteorológicas (Ballesteros, 2014). Los datos predichos de ETo obtenidos a partir del FORETo fueron analizados y comparados con los obtenidos a partir de datos meteorológicos observados utilizando la formula PM-56 como método de referencia.

Para el cálculo de la ETc fue creado un modelo en MATLAB que, utilizando los datos de ETo calculados a través de las dos metodologías presentadas anteriormente y datos de coeficiente de cultivo (Kc) para cada fase de desarrollo del cultivo (Allen et al., 1998) (ECUACIÓN 1), permite calcular 7 días consecutivos de predicción de ETc para todo el ciclo del cultivo.

La duración de las etapas fenológicas para ambos cultivos se estableció mediante observaciones semanales realizadas en campo en las que se registraron aquellos cambios en el grado de cobertura verde y de altura de cultivo correspondientes a las cuatro etapas 
propuestas por Allen et al. (1998) a las cuales se les asignó el Kc propuesto por la misma metodología para cada una de estas etapas.

De esta forma, los resultados obtenidos de ETc, en las dos metodologías, fueron sometidos a los siguientes tratamientos estadísticos: Coeficiente de determinación ajustado $\left(R_{\text {ajust. }}\right.$ ), desviación estándar $(\sigma)$, raíz del error cuadrático medio (RMSE), error relativo $(\varepsilon)$ coeficiente de eficiencia del modelo de Nash-Suthcliff (E) e índice de agregación de Willmott (d). Estos tratamientos fueron hechos utilizando los programas Excel 2010 y Statgraphics Centurion. Se realizaron los análisis para las dos ubicaciones diferenciadas y representativas de La Mancha Oriental y los dos cultivos de trabajo (maíz y cebolla).

\section{3 - Resultados y discusión}

En las Tablas 1 y 2 están presentados los valores del análisis estadístico del modelo de predicción semanal en cada etapa del ciclo fenológico de la cebolla en las estaciones de Pozo Cañada y Tarazona en el año 2011.

Tabla 1. Análisis estadístico de ajuste del modelo de predicción semanal de ETc en la estación de Pozo Cañada por etapas del ciclo fenológico del cultivo de cebolla en el año 2011.

\begin{tabular}{ccccccccccccc}
\hline \multirow{2}{*}{ Día } & \multicolumn{3}{c}{ Etapa 1 } & \multicolumn{3}{c}{ Etapa 2 } & \multicolumn{3}{c}{ Etapa 3 } & \multicolumn{3}{c}{ Etapa 4 } \\
\cline { 2 - 14 } & $\mathrm{R}_{\text {ajust. }}$ & $\mathrm{E}$ & $\mathrm{d}$ & $\mathrm{R}_{\text {ajust. }}^{2}$ & $\mathrm{E}$ & $\mathrm{d}$ & $\mathrm{R}_{\text {ajust. }}$ & $\mathrm{E}$ & $\mathrm{d}$ & $\mathrm{R}_{\text {ajust. }}$ & $\mathrm{E}$ & $\mathrm{d}$ \\
\hline $\mathrm{MD}$ & 0.99 & 0.97 & 0.99 & 0.97 & 0.92 & 0.98 & 0.91 & 0.91 & 0.98 & 0.89 & 0.89 & 0.97 \\
1 & 0.98 & 0.96 & 0.99 & 0.95 & 0.91 & 0.98 & 0.91 & 0.89 & 0.98 & 0.89 & 0.90 & 0.97 \\
2 & 0.97 & 0.95 & 0.99 & 0.92 & 0.86 & 0.97 & 0.92 & 0.90 & 0.98 & 0.84 & 0.87 & 0.97 \\
3 & 0.97 & 0.95 & 0.99 & 0.93 & 0.84 & 0.96 & 0.93 & 0.91 & 0.98 & 0.81 & 0.88 & 0.97 \\
4 & 0.96 & 0.94 & 0.98 & 0.92 & 0.83 & 0.96 & 0.92 & 0.91 & 0.98 & 0.80 & 0.86 & 0.96 \\
5 & 0.96 & 0.94 & 0.98 & 0.90 & 0.78 & 0.95 & 0.91 & 0.89 & 0.98 & 0.78 & 0.85 & 0.96 \\
6 & 0.96 & 0.94 & 0.98 & 0.90 & 0.79 & 0.95 & 0.92 & 0.90 & 0.98 & 0.78 & 0.86 & 0.96 \\
\hline
\end{tabular}

$\mathrm{MD}=$ Mismo día.

Tabla 2. Análisis estadístico de ajuste del modelo de predicción semanal de ETc en la estación de Tarazona por etapas del ciclo fenológico del cultivo de cebolla en el año 2011.

\begin{tabular}{|c|c|c|c|c|c|c|c|c|c|c|c|c|}
\hline \multirow{2}{*}{ Día } & \multicolumn{3}{|c|}{ Etapa 1} & \multicolumn{3}{|c|}{ Etapa 2} & \multicolumn{3}{|c|}{ Etapa 3} & \multicolumn{3}{|c|}{ Etapa 4} \\
\hline & $\mathrm{R}_{\text {ajust. }}^{2}$. & $E$ & d & $\mathrm{R}_{\text {aiust. }}^{2}$ & $E$ & $d$ & $\mathrm{R}^{2}$ ajust. & $E$ & $\mathrm{~d}$ & $R_{\text {ajust. }}^{2}$ & $E$ & d \\
\hline MD & 0.95 & 0.94 & 0.98 & 0.05 & -0.78 & 0.56 & 0.86 & 0.86 & 0.96 & 0.70 & 0.67 & 0.87 \\
\hline 1 & 0 & 0.94 & .9 & 0.0 & -0.78 & 0.5 & 0.8 & 0.87 & 0 & 0 & .70 & 0.89 \\
\hline 2 & 0.95 & 0.94 & 0.98 & 0.05 & -0.90 & 0.54 & 0.87 & 0.87 & 0.96 & 0.71 & 0.71 & 0.90 \\
\hline 3 & 0 & 0.94 & 0.98 & 0.05 & -1.07 & 0.55 & 0.8 & 0.87 & 0 & 3 & 0.70 & 0.88 \\
\hline 4 & & 0.95 & 0.98 & & 8 & 0 & 0 & 0.91 & 0 & & 30 & 0.92 \\
\hline 5 & 0.95 & 0.95 & 0.98 & 0.06 & -1.28 & 0.54 & 0.90 & 0.91 & 0.97 & 0.82 & 0.80 & 0.92 \\
\hline 6 & 0.96 & 0.95 & 0.99 & 0.04 & -1.17 & 0.53 & 0.90 & 0.91 & 0.98 & 0.80 & 0.81 & 0.93 \\
\hline
\end{tabular}

$\mathrm{MD}=$ Mismo día.

Es posible observar que, en la estación de Pozo Cañada, el modelo se ajusta bien a todas las etapas de desarrollo del cultivo. En la estación de Tarazona, en la etapa 2, el modelo no presentó buen ajuste, lo que puede ser debido a un evento de lluvia que ocurrió en esta etapa. En el periodo llovió una cantidad acumulada de $39 \mathrm{~mm}$, que fueron distribuidas en dos eventos de mayor cantidad (17.2 y $11.2 \mathrm{~mm}$ ) y otras lluvias de pequeña intensidad. Pero, a pesar de una cantidad acumulada pequeña, el facto de haber llovido en algunos días y la pequeña duración de la etapa hicieron con que el ajuste bajase considerablemente. En las otras etapas el modelo presentó valores de ajuste dentro del rango aceptable. Se nota también un comportamiento común a la mayoría de los resultados presentados anteriormente, que es lo de disminuir el ajuste a medida en que se van aumentando los días de predicción, o sea, la predicción para el mismo día, normalmente, tiene mejor ajuste do que las predicciones para los 7 siguientes. 
En las Tablas 3 y 4 se puede observar los valores del análisis estadístico del modelo de predicción semanal en cada etapa del ciclo fenológico de la cebolla en las estaciones de Pozo Cañada y Tarazona en el año 2012.

Tabla 3. Análisis estadístico de ajuste del modelo de predicción semanal de ETc en la estación de Pozo Cañada por etapas del ciclo fenológico del cultivo de cebolla en el año 2012.

\begin{tabular}{cccccccccccccc}
\hline \multirow{2}{*}{ Día } & \multicolumn{3}{c}{ Etapa 1 } & \multicolumn{3}{c}{ Etapa 2 } & \multicolumn{3}{c}{ Etapa 3 } & \multicolumn{3}{c}{ Etapa 4 } \\
\cline { 2 - 15 } & $\mathrm{R}_{\text {ajust. }}^{2}$ & $\mathrm{E}$ & $\mathrm{d}$ & $\mathrm{R}_{\text {ajust. }}$ & $\mathrm{E}$ & $\mathrm{d}$ & $\mathrm{R}_{\text {ajust. }}$ & $\mathrm{E}$ & $\mathrm{d}$ & $\mathrm{R}_{\text {ajust. }}$ & $\mathrm{E}$ & $\mathrm{d}$ \\
\hline $\mathrm{MD}$ & 0.99 & 0.99 & 1.00 & 0.85 & 0.85 & 0.96 & 0.91 & 0.87 & 0.97 & 0.74 & 0.12 & 0.86 \\
1 & 0.98 & 0.98 & 0.99 & 0.85 & 0.83 & 0.96 & 0.85 & 0.80 & 0.96 & 0.73 & 0.07 & 0.86 \\
2 & 0.97 & 0.97 & 0.99 & 0.82 & 0.78 & 0.95 & 0.82 & 0.77 & 0.95 & 0.70 & -0.06 & 0.85 \\
3 & 0.96 & 0.96 & 0.99 & 0.77 & 0.74 & 0.94 & 0.83 & 0.78 & 0.95 & 0.71 & -0.17 & 0.84 \\
4 & 0.96 & 0.96 & 0.99 & 0.73 & 0.70 & 0.92 & 0.86 & 0.81 & 0.96 & 0.73 & -0.15 & 0.85 \\
5 & 0.96 & 0.96 & 0.99 & 0.72 & 0.68 & 0.92 & 0.89 & 0.84 & 0.97 & 0.74 & -0.10 & 0.86 \\
6 & 0.96 & 0.95 & 0.99 & 0.70 & 0.62 & 0.91 & 0.90 & 0.86 & 0.97 & 0.75 & 0.07 & 0.87 \\
\hline
\end{tabular}

$\mathrm{MD}=$ Mismo día.

Tabla 4. Análisis estadístico de ajuste del modelo de predicción semanal de ETc en la estación de Tarazona por etapas del ciclo fenológico del cultivo de cebolla en el año 2012.

\begin{tabular}{cccccccccccccc}
\hline \multirow{2}{*}{ Día } & \multicolumn{3}{c}{ Etapa 1 } & \multicolumn{4}{c}{ Etapa 2 } & \multicolumn{3}{c}{ Etapa 3 } & \multicolumn{3}{c}{ Etapa 4 } \\
\cline { 2 - 15 } & $\mathrm{R}_{\text {aiust. }}^{2} \mathrm{E}$ & $\mathrm{d}$ & $\mathrm{R}_{\text {aiust. }}$ & $\mathrm{E}$ & $\mathrm{d}$ & $\mathrm{R}_{\text {ajust. }}$ & $\mathrm{E}$ & $\mathrm{d}$ & $\mathrm{R}_{\text {ajust. }}$ & $\mathrm{E}$ & $\mathrm{d}$ \\
\hline $\mathrm{MD}$ & 0.96 & 0.96 & 0.99 & 0.86 & 0.87 & 0.96 & 0.96 & 0.95 & 0.99 & 0.82 & 0.29 & 0.90 \\
1 & 0.93 & 0.93 & 0.98 & 0.81 & 0.82 & 0.95 & 0.94 & 0.94 & 0.98 & 0.83 & 0.41 & 0.91 \\
2 & 0.89 & 0.89 & 0.97 & 0.77 & 0.78 & 0.94 & 0.95 & 0.94 & 0.99 & 0.83 & 0.40 & 0.91 \\
3 & 0.87 & 0.87 & 0.97 & 0.78 & 0.79 & 0.94 & 0.95 & 0.94 & 0.99 & 0.84 & 0.40 & 0.91 \\
4 & 0.87 & 0.88 & 0.97 & 0.80 & 0.80 & 0.95 & 0.96 & 0.95 & 0.99 & 0.86 & 0.40 & 0.91 \\
5 & 0.87 & 0.87 & 0.97 & 0.80 & 0.80 & 0.95 & 0.96 & 0.95 & 0.99 & 0.84 & 0.36 & 0.90 \\
6 & 0.87 & 0.87 & 0.97 & 0.77 & 0.77 & 0.94 & 0.96 & 0.95 & 0.99 & 0.86 & 0.36 & 0.91 \\
\hline
\end{tabular}

$\mathrm{MD}=$ Mismo día.

Se nota que el modelo no se ajusta bien en la última etapa de cultivo en la estación de Pozo Cañada, pero se muestra muy bien ajustado en las 3 etapas iniciales. Los valores presentados en la etapa 4 nos dicen que el modelo presenta valores de predicción, durante la etapa, parecidos con los de la media de las observaciones en el período (TABLA 3). Eso puede ter ocurrido por el facto de que en la última etapa la cebolla baja su ETc muy rápidamente, en cuanto que en las predicciones esa disminución va un poco más despacio, ocasionando ese ligero desajuste en el modelo. Lo mismo ocurre en la estación de Tarazona (TABLA 4), siendo que en esta estación el ajuste se presenta un poco mejor do que en Pozo Cañada.

En la Figura 1, Estación de Pozo Cañada, se puede ver la ETc acumulada estimada por predicciones semanales por las dos metodologías (PM-56 y RNA). Se nota que los valores predichos siguen la misma tendencia de los valores observados, o sea, cuando aumentan los valores de ETc estimados por PM-56, aumentan también los estimados por las RNA, aún que no en la misma cantidad. Se observa también que en el año 2012 el modelo se ajusta mejor do que el año 2011 hasta un poco más de la mitad del ciclo, disminuyendo el ajuste en el final del ciclo. 

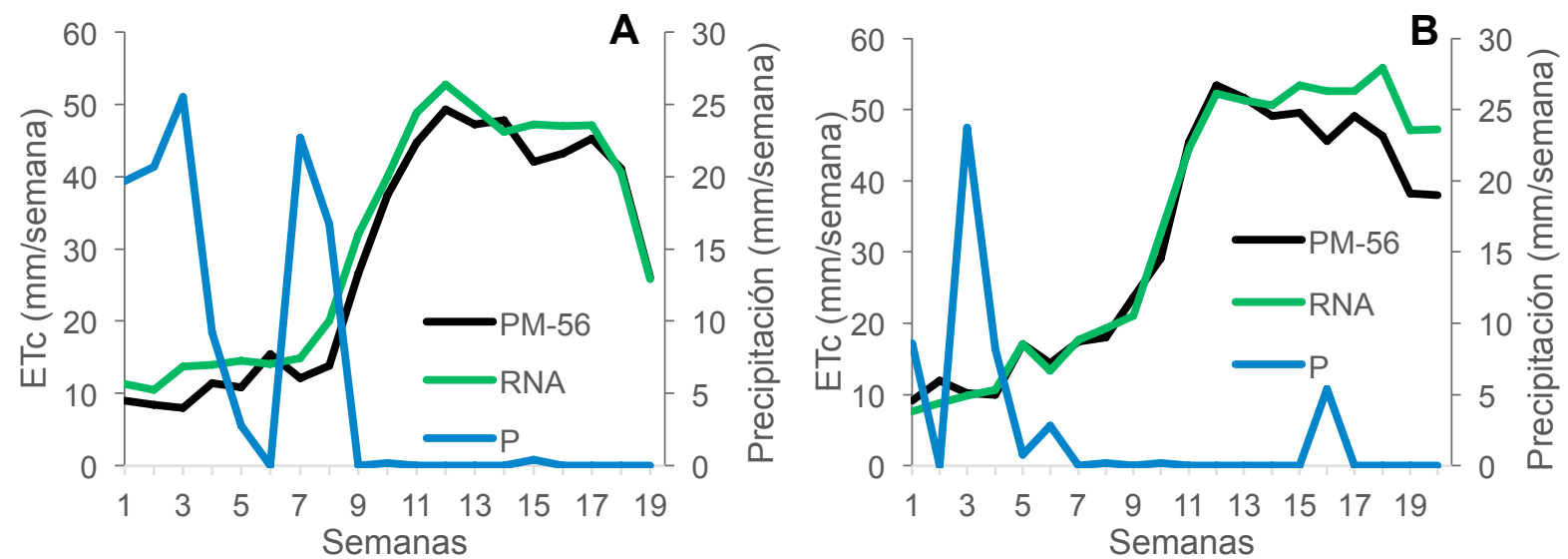

Figura 1. ETc observada (PM-56) versus ETc predicha (RNA) en la Estación Meteorológica de Pozo Cañada para los años de 2011 (A) y 2012 (B) en el cultivo de cebolla.

Lo mismo ocurre en la estación de Tarazona (FIGURA 2), ya que en los dos años las predicciones siguen la misma tendencia de las observaciones. En la comparación mientras los dos años en Tarazona se percibe un mejor ajuste en el año 2012. Se nota que en el inicio del ciclo la gran cantidad de lluvia no afecta mucho el ajuste del modelo. Pero en el final del ciclo, una ligera lluvia hace con que el modelo sobrestime las observaciones (FIGURA 2B).
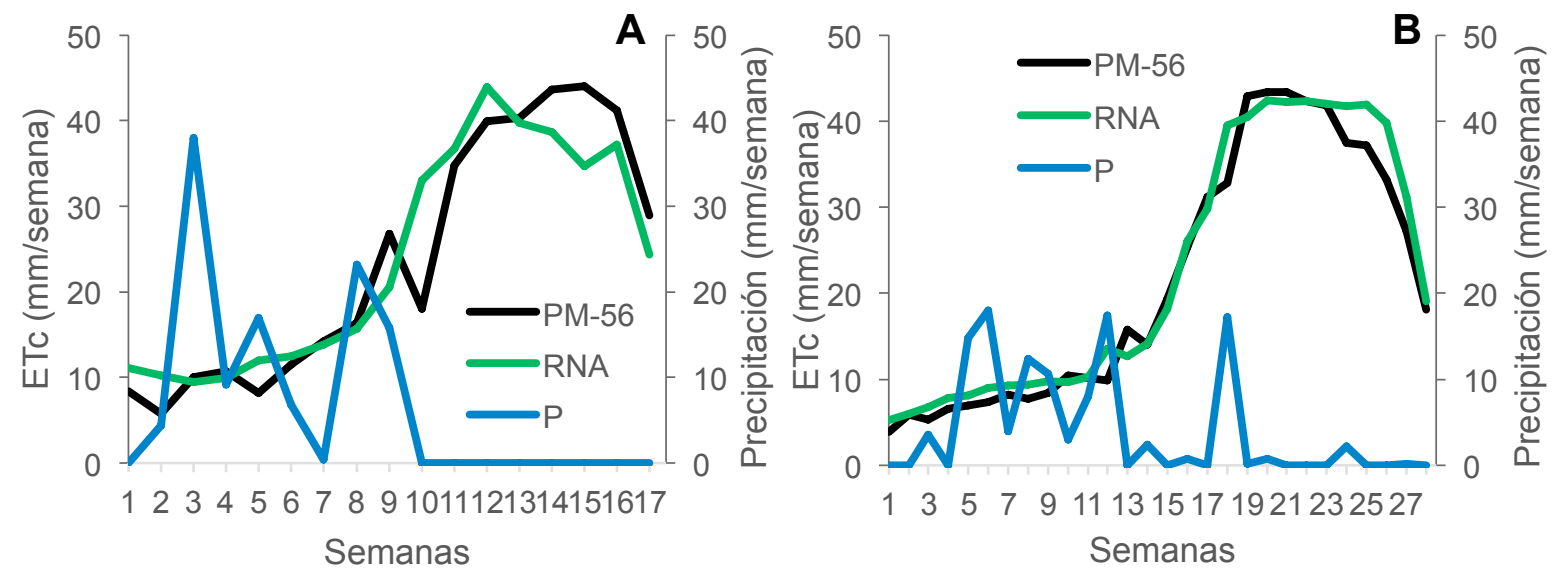

Figura 2. ETc observada (PM-56) versus ETc predicha (RNA) en la Estación Meteorológica de Tarazona para los años de 2011 (A) y 2012 (B) en el cultivo de cebolla.

Fueron hechas también comparaciones mientras los dos cultivos. Para esta comparación fueron utilizados datos de ETc calculados para el cultivo de maíz para los años de 2011 y 2012 en la estación de Tarazona (TABLAS 5 Y 6). Así se puede comparar los resultados del maíz con los de cebolla en la misma estación, presentados en las Tablas 3 y 4.

Tabla 5. Análisis estadístico de ajuste del modelo de predicción semanal de ETc en la estación de Tarazona por etapas del ciclo fenológico del cultivo de maíz en el año 2011.

\begin{tabular}{cccccccccccccc}
\hline \multirow{2}{*}{ Día } & \multicolumn{3}{c}{ Etapa 1 } & \multicolumn{3}{c}{ Etapa 2 } & \multicolumn{3}{c}{ Etapa 3 } & \multicolumn{3}{c}{ Etapa 4 } \\
\cline { 2 - 14 } & $\mathrm{R}_{\text {ajust. }}^{2} \mathrm{E}$ & $\mathrm{d}$ & $\mathrm{R}_{\text {ajust. }}$ & $\mathrm{E}$ & $\mathrm{d}$ & $\mathrm{R}_{\text {ajust. }}$ & $\mathrm{E}$ & $\mathrm{d}$ & $\mathrm{R}_{\text {ajust. }}$ & $\mathrm{E}$ & $\mathrm{d}$ \\
\hline $\mathrm{MD}$ & 0.88 & 0.88 & 0.97 & 0.71 & 0.72 & 0.91 & 0.85 & 0.82 & 0.94 & 0.77 & 0.78 & 0.93 \\
1 & 0.88 & 0.88 & 0.97 & 0.73 & 0.73 & 0.92 & 0.85 & 0.83 & 0.94 & 0.77 & 0.77 & 0.93 \\
2 & 0.88 & 0.88 & 0.97 & 0.71 & 0.71 & 0.91 & 0.86 & 0.83 & 0.95 & 0.79 & 0.79 & 0.94 \\
3 & 0.87 & 0.88 & 0.97 & 0.69 & 0.69 & 0.89 & 0.85 & 0.83 & 0.94 & 0.81 & 0.81 & 0.94 \\
4 & 0.87 & 0.87 & 0.96 & 0.71 & 0.69 & 0.89 & 0.91 & 0.89 & 0.96 & 0.80 & 0.80 & 0.94 \\
5 & 0.87 & 0.87 & 0.96 & 0.72 & 0.67 & 0.88 & 0.90 & 0.88 & 0.96 & 0.79 & 0.79 & 0.94 \\
6 & 0.88 & 0.88 & 0.96 & 0.73 & 0.68 & 0.88 & 0.91 & 0.89 & 0.97 & 0.80 & 0.80 & 0.94 \\
\hline
\end{tabular}


Tabla 6. Análisis estadístico de ajuste del modelo de predicción semanal de ETc en la estación de Tarazona por etapas del ciclo fenológico del cultivo de maíz en el año 2012.

\begin{tabular}{|c|c|c|c|c|c|c|c|c|c|c|c|c|}
\hline \multirow{2}{*}{ Día } & \multicolumn{3}{|c|}{ Etapa 1} & \multicolumn{3}{|c|}{ Etapa 2} & \multicolumn{3}{|c|}{ Etapa 3} & \multicolumn{3}{|c|}{ Etapa 4} \\
\hline & $\mathrm{R}_{\text {ajust. }}^{2}$ & $E$ & $\mathrm{~d}$ & $\mathrm{R}_{\text {ajust. }}^{2}$ & $E$ & $\mathrm{~d}$ & $\mathrm{R}_{\text {ajust. }}^{2}$ & $E$ & d & $\mathrm{R}_{\text {ajust. }}^{2}$ & $E$ & d \\
\hline MD & 0.98 & 0.97 & 0.99 & 0.83 & 0.86 & 0.96 & 0.93 & 0.92 & 0.98 & 0.91 & 0.86 & 0.96 \\
\hline 1 & 0.97 & 0.95 & 0.99 & 0.77 & 0.81 & 0.94 & 0.91 & 0.89 & 0.98 & 0.91 & 0.86 & 0.96 \\
\hline 2 & 0.94 & 0.92 & 0.98 & 0.74 & 0.78 & 0.94 & 0.91 & 0.89 & 0.98 & 0.91 & 0.86 & 0.96 \\
\hline 3 & 0.94 & 0.91 & 0.98 & 0.77 & 0.80 & 0.94 & 0.92 & 0.89 & 0.98 & 0.91 & 0.87 & 0.96 \\
\hline 4 & 0.94 & 0.91 & 0.98 & 0.81 & 0.81 & 0.81 & 0.93 & 0.90 & 0.98 & 0.91 & 0.86 & 0.96 \\
\hline 5 & 0.94 & 0.92 & 0.98 & 0.81 & 0.83 & 0.95 & 0.94 & 0.92 & 0.98 & 0.91 & 0.86 & 0.96 \\
\hline 6 & 0.94 & 0.91 & 0.98 & 0.78 & 0.80 & 0.94 & 0.94 & 0.92 & 0.98 & 0.91 & 0.87 & 0.97 \\
\hline
\end{tabular}

$\mathrm{MD}=$ Mismo día.

Ahí se puede observar que el modelo también mantiene un buen ajuste para los 7 días de predicción en todas las etapas para el año 2011, presentando una ligera reducción del ajuste en la etapa 2. Ya en el año 2012, eventos de lluvia en la etapa 2 hicieron con que el modelo redujese su ajuste, reducción esta que puede ter sido ocasionada por dos lluvias con registros de 10.8 y $7 \mathrm{~mm}$, totalizando $18 \mathrm{~mm}$ de lluvia en el período. En las etapas 1,3 y 4 el modelo se presentó muy bien ajustado a los valores observados en la estación meteorológica.

A partir de ahí se ha podido hacer una comparación mientras los dos cultivos. En la Figura 3 se puede observar la ETc del cultivo de maíz en la estación de Tarazona para los años 2011 y 2012.
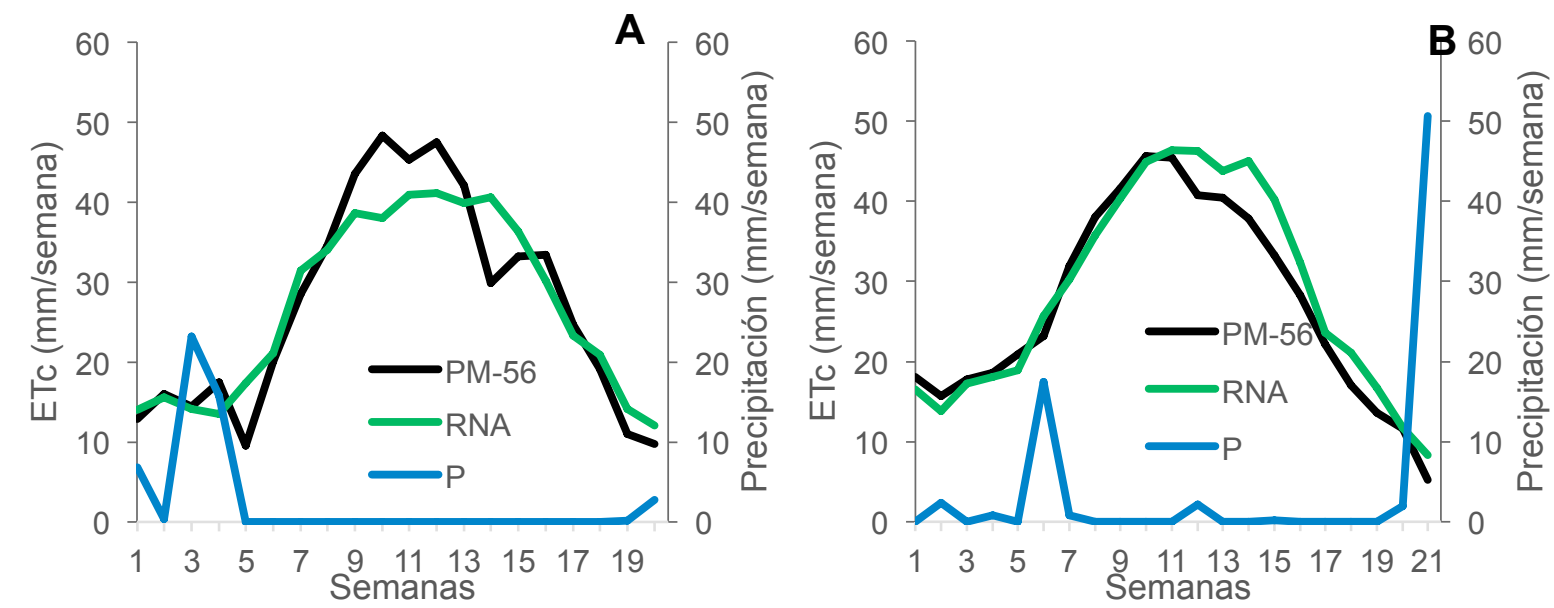

Figura 3. ETc observada (PM-56) versus ETc predicha (RNA) en la Estación Meteorológica de Tarazona para los años de 2011 (A) y 2012 (B) en el cultivo de maíz.

En la comparación mientras los cultivos se puede observar que el modelo se ajusta mejor al cultivo de maíz en las etapas 1 y 3 , en cuanto en las etapas 2 y 4 el mejor ajuste se da en el cultivo de la cebolla.

Merece la pena resaltar que todos los modelos presentados sufrieron interferencia por efecto de la lluvia, lo que es común en los modelos de predicción de las necesidades de riegos de los cultivos. 


\section{4 - Conclusiones y recomendaciones}

Las predicciones presentan un buen ajuste en relación a los datos observados en las estaciones, lo que demuestra que el modelo puede ser utilizado como una herramienta adicional de ayuda a los regantes, que podrán tener una predicción de 7 días con una gran fiabilidad de los datos.

El modelo se ajusta muy bien a las etapas iniciales del maíz, presentando siempre valores de $\mathrm{R}^{2}$ superiores a 0.9 .

Eventos de lluvia afectan considerablemente los modelos de predicción de ETo.

El modelo ofrece una oportunidad para que estas herramientas sirvan como mejora en la calidad de gestión de los recursos hídricos en España.

\section{5 - Agradecimientos}

Agradezco al Proyecto IBrasil - Erasmus Mundus por financiar mis estudios de doctorado en la Universidad de Castilla-La Mancha.

\section{6 - Referencias}

AEMET (2014). Datos de predicción y el tiempo por municipios. <http://www.aemet.es/es/eltiempo/prediccion> Desde Septiembre/2014 hasta Febrero/2015.

Allen, R.G., Pereira, L.S., Raes, D., Smith, M. (1998). Crop evapotranspiration. Guidelines for computing crop water requirements FAO Irrigation and drainage paper No 56. Food and Agriculture Organization (FAO). Rome.

Ballesteros González, R. 2014. Predicción de evapotranspiración de referencia y empleo de modelos de tiempo térmico junto a información geomática en la caracterización de cultivos para la gestión del regadío en la Mancha Oriental. Tesis Doctoral. Escuela Técnica Superior de Ingenieros Agrónomos, Universidad de Castilla La Mancha, Albacete, España.

Comprehensive Assessment of Water Management in Agriculture. 2007. Water for Food, Water for Life: A Comprehensive Assessment of Water Management in Agriculture. London: Earthscan, and Colombo: International Water Management Institute.

ECMWF (2015). Forecast Datasets. < http://www.ecmwf.int/en/forecasts/datasets> Enero/2015.

MARM (2010). Estrategia nacional para la modernización sostenible H2015. Informe de sostenibilidad ambiental. (National strategy for sustainable irrigation modernization H2015. Environmental sustainability report). Resource document. Ministerio de Medio Ambiente, Medio Rural y Marino.

$<$ http://www.magrama.gob.es/es/calidad-yevaluacionambiental/participacionpublica/2isa_eae_enmsrh2015_210710_parte1_tcm7111305.pdf.> Febrero/2015. 